\title{
FDA-approved Antitussive Dextromethorphan Enhances Antifibrotic Effectiveness of Pirfenidone in Bleomycin-induced Mice and Patients with Idiopathic Pulmonary Fibrosis
}

Nana Liu

Tianjin Medical University General Hospital

\section{Yubao Wang}

The Second Hospital of Tianjin Medical University

Jie Huang

Tianjin Medical University General Hospital

\section{Yunze Du}

Tianjin People's Hospital

\section{Luqing Wei}

Tianjin Beichen Hospital

\section{Dongsheng Li}

Tianjin Nankai Hospital

\section{Jau-Shyong Hong}

National Institutes of Health

\section{Wen Ning}

Nankai University

Jing Feng ( $\nabla$ fengjing1974@163.com )

Tianjin Medical University General Hospital

\section{Research Article}

Keywords: Idiopathic pulmonary fibrosis, Pirfenidone, Dextromethorphan, Bleomycin, Pulmonary function testing, High resolution computed tomography, HRCT

Posted Date: August 6th, 2021

DOI: https://doi.org/10.21203/rs.3.rs-472298/v2

License: (c) (i) This work is licensed under a Creative Commons Attribution 4.0 International License. Read Full License 


\section{Abstract}

Background: We aimed to investigate whether dextromethorphan (DM), an antitussive agent, could improve the antifibrotic efficacy of pirfenidone in treating idiopathic pulmonary fibrosis, a fatal interstitial lung disease characterized by progressive and irreversible respiratory failure.

Methods: A bleomycin-induced mouse pulmonary fibrosis model study and an open-label randomized clinical trial were performed to evaluate the effectiveness of pirfenidone combined with DM.

Results: In the animal study, pirfenidone combined with DM protected the mice against bleomycininduced pulmonary fibrosis with better capabilities than pirfenidone or DM alone, as indicated by lung histologic analysis and hydroxyproline levels. In the clinical study, pirfenidone plus DM markedly mitigated pulmonary function (FEV1 and FVC) decline and ameliorated chest HRCT imaging scores (ground glass opacities and reticulation) of patients with IPF compared with pirfenidone alone at one year after administration. There were no significant differences in adverse reactions between the pirfenidone-DM group and the pirfenidone group.

Conclusions: Pirfenidone plus DM may be a better strategy to modify IPF than pirfenidone alone. The efficacy and safety of the combination of pirfenidone and DM for patients with IPF warrants further verification by a double-blind randomized controlled trial (RCT).

\section{Background}

Idiopathic pulmonary fibrosis (IPF) is a chronic, progressive, and fatal interstitial lung disease (ILD) of unknown aetiology. It is characterized by the destruction of the alveolar structure and abnormal deposition of extracellular matrix in the alveolar cavity and lung interstitium. Patients with IPF develop chest tightness, shortness of breath, and ultimately, respiratory failure. The median survival time after diagnosis of IPF is only 2-3 years [1]. At present, disease progression cannot be completely reversed unless lung transplantation is performed [2].

Many factors related to inflammation, such as interferon- $\triangle$ (IFN- $\varnothing$ ), interleukin (IL)-4, transforming growth factor- $\beta$ (TGF- $\beta$ ), a-SMA, and oxidative stress, have been found to be involved in the pathogenesis of IPF, leading to the abnormal proliferation of fibroblasts and myofibroblasts and the aberrant deposition of extracellular matrix in the lung [3]. Decreased IFN- $\triangle$ and increased IL-4 promote fibroblast proliferation and collagen deposition [4]. TGF- $\beta$ initiates epithelial-mesenchymal transition (EMT) of alveolar epithelial cells by enhancing the production of mitochondrial reactive oxidative species (ROS) [5]. Myofibroblasts expressing a-SMA cannot undergo normal apoptosis. Oxidative stress can damage epithelial cells, upregulate the expression of a variety of profibrotic mediators and cytokines, and cause a lung cathepsin and anti-protease imbalance, eventually resulting in a large amount of extracellular matrix deposition and the formation of pulmonary fibrosis. Therefore, ROS-generating NADPH oxidases (NOX), including Nox1, Nox2, and Nox4, play critical roles in the pathogenesis of IPF. For example, genetic ablation of the p47phox subunit of Nox2 protected mice against bleomycin-induced lung fibrosis [6]. 
Pirfenidone, a TGF- $\beta$ synthesis inhibitor with anti-inflammatory, antioxidant and antifibrotic effects, was approved in 2014 by the Food and Drug Administration (FDA) to treat IPF [7]. It reduces the accumulation of hydroxyproline, the expression of smooth actin (a-SMA), collagen deposition, and fibroblast proliferation by inhibiting the TGF- $\beta 1 /$ Smad and PI3K/Akt signalling pathways. The 2015 ATS/ERS/JRS/ALAT guidelines conditionally recommend pirfenidone for the treatment of patients with IPF [8]. Although pirfenidone improves patients' symptoms such as dyspnoea, it only moderately slows pulmonary functional decline and disease progression [9]. How to improve the therapeutic effect of IPF is a formidable challenge.

Dextromethorphan (DM), a dextrorotatory enantiomer of the opioid agonist levorphanol, is a widely used over-the-counter antitussive drug approved by the FDA [10]. In recent years, the inflammation-repression effects of DM have been gradually recognized and discovered. DM reduces serum levels of the proinflammatory mediators tumour necrosis factor alpha (TNF-a), IL-6, IL-1 chemokine monocyte chemotactic protein 1 (MCP-1), and macrophage inflammatory protein 2 (MIP-2) in mice or patients [1113]. Low-dose DM inhibits the expression and activity of microglial NOX2 [14]. The anti-inflammatory and antioxidant properties of DM imply that it could be applied to promote the antifibrotic capacity of pirfenidone for IPF.

Here, we found that pirfenidone combined with DM showed the best potency in reducing fibrotic pathology compared with pirfenidone alone or DM alone in a bleomycin (BLM)-induced pulmonary fibrosis mouse model. A subsequent open-label clinical trial showed that pirfenidone plus low-dose DM significantly mitigated pulmonary functional decline and ameliorated pulmonary imaging scores of patients with IPF compared with pirfenidone alone. In addition, DM add-on therapy did not increase the side effects of pirfenidone.

\section{Methods}

\subsection{Preclinical study}

\subsubsection{Mouse model}

C57BL/6J mice (Vital River Laboratory Animal Technology Co., Ltd., Beijing, China) were obtained (male, $8 \sim 9$ weeks old, average weight $23 \sim 25 \mathrm{~g}$ ). They were hosted and bred in a specific pathogen-free environment with access to food and water ad libitum.

\subsubsection{Lung fibrosis model and drug treatment}

The mice were divided into 5 groups of 8 animals each: control group, bleomycin group $(\mathrm{BLM}+\mathrm{NaCl})$, bleomycin + DM group (BLM + DM), bleomycin + pirfenidone group (BLM + PFD), and bleomycin + pirfenidone + DM group (BLM + PFD + DM). The bleomycin 1.5U (1.5 mg/kg) (Nippon Kayaku Co., Tokyo, 
Japan) was intratracheally administered to the mice except for the control group at day 0 . Mice in the different groups were pretreated with vehicle, pirfenidone alone, DM alone, or pirfenidone plus DM for one day and continuously administered until day 20. Pirfenidone (Beijing Kangdini Pharmaceutical Co., Ltd., China) was intragastrically administered once a day $(100 \mathrm{mg} / \mathrm{kg}$ body weight). DM (Selleck Biotechnology Co., Ltd., USA) was subcutaneously injected three times a day ( $10 \mathrm{ng} / \mathrm{kg}$ body weight). The control group and the bleomycin group were given the same volume of saline every day. The mice were sacrificed on D21, and the lungs were removed to prepare for histological staining and hydroxyproline assays.

\subsubsection{Histologic analysis}

The mouse left lungs were dehydrated, paraffin-embedded, cut into $5-\mu \mathrm{m}$ sections, and then stained with H\&E and Masson's trichrome. The degree of inflammation and fibrosis of the lung tissue was observed under an optical microscope. The pulmonary fibrosis area was quantified[15]. The slides were sampled with a random-start systematic sampling scheme using a 6-mm grid randomly superimposed on the slide to indicate the areas to evaluate. Digitized images of the slide were obtained using a $\times 2$ objective on an Olympus BH-2 microscope (Olympus America, Melville, New York, USA) with a Sony DXC970 MD camera (Sony America, New York, New York, USA) and an IxTV capture card (IxMicro, San Jose, California, USA) on an Apple Macintosh G3 computer (Apple Computer Inc., Cupertino, California, USA). The images were then opened in Photoshop Elements (Adobe Systems Inc., San Jose, California, USA). The overall area of the lung was obtained by manual outlining. The area of the lung with fibrosis was then outlined, and the area was obtained. The pixels of the total versus fibrotic tissue were then summed over each lung, and a percentage was obtained.

\subsubsection{Hydroxyproline assay}

The collagen contents were evaluated with the conventional hydroxyproline method in the mouse right lungs after they had been cleared of blood [16]. Samples containing known amounts of purified collagen confirmed the capability of the assay to fully hydrolyse and recover hydroxyproline from collagen.

\subsection{Human study}

\subsubsection{Patients}

This study was a multicentre, open-label, randomized study conducted by the Tianjin Medical University General Hospital, Tianjin Academy of Traditional Chinese Medicine Affiliated Hospital, Tianjin People's Hospital, and Tianjin Beichen Hospital. This study was approved by the Ethics Committee of Tianjin Medical University General Hospital, China (No. IRB2020-YX-031-01). Informed consent was obtained 
from all study participants. All consecutive patients who had started pirfenidone treatment between May 2019 and December 2020 were candidates for our study. Patients were selected based on the following criteria: (1) The diagnosis of IPF was made in accordance with the 2018 ATS/ERS/JRS/ALAT guidelines [17], and the patient's chest HRCT conformed or possibly conformed to the UIP pattern. (2) Glucocorticoids or immunosuppressants were not administered within 1 month. All patients took pirfenidone capsules (Beijing Kangdini Pharmaceutical Co., Ltd., 100 mg/capsule, H20133376). An initial dose of $200 \mathrm{mg}$ of pirfenidone was administered three times daily with meals ( $600 \mathrm{mg} /$ day). Thereafter, the dose was gradually increased by $200 \mathrm{mg}$ every 2 weeks to a maximum of $600 \mathrm{mg}$ per dose (1800 $\mathrm{mg} /$ day). Dextromethorphan (DM) hydrobromide tablets (Shijiazhuang Yiling Pharmaceutical Co., Ltd., $15 \mathrm{mg} /$ tablet, $\mathrm{H} 20066348$ ) were added at $7.5-15 \mathrm{mg}$ once a day for the pirfenidone-DM group. A total of 16 consecutive eligible patients were enrolled, with 8 in the pirfenidone group and 8 in the pirfenidone-DM group.

\subsubsection{Laboratory tests and pulmonary function testing}

Routine blood tests, C-reactive protein (CRP), liver and kidney function tests, and pulmonary function tests (PFTs) of the patients were collected before treatment, and at 6 months and 1 year after starting treatment. Pulmonary function tests (PFTs) were performed by standard techniques using CHESTAC-33 (Chest MI Co., Tokyo, Japan) and Fudac-77 (Fukuda Denshi, Tokyo). Pulmonary function test indexes included forced vital capacity (FVC), forced vital capacity percent (FVC\%) predicted, forced expiratory volume in one second (FEV1), forced expiratory volume in one second percent (FEV1\%) predicted and the diffusing capacity of the lung for carbon monoxide percent (DLCO\%) predicted.

\subsubsection{CT images}

A high-resolution CT scan (Lightspeed-64, GE, America) was used, and CT images with $0.5 \mathrm{~mm}$ (or $1 \mathrm{~mm}$ ) slice thickness were obtained (140 kVp and $200 \mathrm{~mA}$ ). Each extent of normal lung and each lesion (ground glass opacities (GGOs), consolidation, reticular abnormality, honeycombing and emphysema) was recorded at $5 \%$ steps in three zones in each lung based on the method described by Best [18]. The upper zone, middle zone, and lower zone were defined as at or above the aortic arch, between the aortic arch and the pulmonary veins, and at or below the pulmonary veins, respectively. The mean extent of the three zones was calculated. The HRCT scans were scored by two expert thoracic radiologists using the above method, which assesses the degree of ground glass attenuation (HRCT alveolar score) and fibrotic change (HRCT interstitial score and honeycomb score) [19]. Radiologists were blinded to the patient's diagnosis and grouping. The agreement between the radiologists was very good.

\subsection{Statistical analysis}


Statistical analyses were performed using GraphPad Prism 7 (GraphPad Software, La Jolla, California, USA). Multiple comparisons were performed using ANOVA tests with post hoc analysis. To compare the demographic data and baseline clinical characteristics between the pirfenidone group and the pirfenidone-DM group, a chi-square test and Fisher's exact test for categorical variables and a MannWhitney $\mathrm{U}$ test for continuous variables were used as appropriate. The level of interobserver agreement between the two radiologists was evaluated by kappa statistic measurements. $P$ values $<0.05$ were considered to indicate statistical significance.

\section{Results}

\subsection{Pirfenidone combined with DM protects mice against bleomycin-induced pulmonary fibrosis}

At 21 days after challenge, intratracheal injection of bleomycin resulted in the destruction of the normal lung structures, markedly thickened alveolar septum, and mass filling of the fibrous tissue as indicated by H\&E staining of the mouse lung sections (Fig. 1A). The deposition of collagen fibres was increased in the bleomycin-induced lung injury, as shown by Masson's trichrome staining (Fig. 1A). Decreased alveolar damage and collagen fibre deposition were displayed in the mouse lung sections by the administration of pirfenidone, DM, or pirfenidone combined with DM (Fig. 1A). The fibrosis focal area ratio by quantitative analysis in the four groups (BLM + NaCl, BLM + DM, BLM + PFD, and BLM + PFD + DM) was $0.097 \pm 0.019$, $0.058 \pm 0.047,0.056 \pm 0.043$, and $0.031 \pm 0.034$ (mean $\pm S D$ ), respectively. Among these four groups, pirfenidone combined with DM significantly reduced the fibrosis focal area ratio caused by bleomycin. The lung fibrosis lesion area ratio of mice in the BLM + PFD + DM group was reduced significantly compared with that in either the BLM + PFD group or the BLM + DM group $(P<0.05)$ (Fig. 1B).

Hydroxyproline (HPO), a nonessential amino acid found in collagen, plays a crucial role in collagen synthesis and serves as a biochemical marker of lung fibrosis. At day 21 after exposure to bleomycin or vehicle, the lung hydroxyproline contents in the five groups (control, $\mathrm{BLM}+\mathrm{NaCl}, \mathrm{BLM}+\mathrm{DM}, \mathrm{BLM}+\mathrm{PFD}$, and BLM + PFD + DM) were 209.2 $\pm 23.5,400.1 \pm 63.6,346.3 \pm 59.3,317.7 \pm 30.1$, and $302.7 \pm 49.3$ $\mu \mathrm{g} /$ lung (mean $\pm S D$ ), respectively. The levels of lung hydroxyproline in the BLM + DM group, BLM + PFD group and BLM + PFD + DM group were significantly lower than those in the bleomycin group. The levels of lung hydroxyproline in the BLM + PFD + DM group were lower than those in the BLM + PFD group or $B L M+D M$ group $(P<0.05)$ (Fig. 1C). Together, the fibrosis focal area ratio quantification and hydroxyproline assay clearly indicated the potency of pirfenidone plus DM in alleviating bleomycinelicited pulmonary fibrotic pathology.

\subsection{Patient characteristics}


Based on the findings of the mouse experiments, we then studied whether DM could potentiate the treatment effectiveness of pirfenidone in patients with IPF. We enrolled 20 patients, but 4 patients ( 2 from the pirfenidone group and 2 from the pirfenidone-DM group) died of infections. Therefore, the PFTs and HRCT scores of 16 patients were statistically analysed. There were no significant differences in sex, age, age at diagnosis, clinical-radiological diagnosis, smoking years, prior treatment with steroids, or IPF treatment at baseline (Table 1 ). Three patients (50.00\%) in the pirfenidone group and two patients (37.50\%) in the pirfenidone-DM group showed the usual interstitial pneumonia (UIP) pattern on HRCT, but there was no significant difference between the two groups (Table 1).

Table 1

Patients demographics and clinical characteristics.

\begin{tabular}{|c|c|c|c|}
\hline Characteristics & Pirfenidone $(\mathrm{N}=8)$ & Pirfenidone + DM $(\mathrm{N}=8)$ & $P^{*}$ \\
\hline Female-n (\%) & $2(25)$ & $3(38)$ & .590 \\
\hline$\nabla 60-n(\%)$ & $5(63)$ & $6(75)$ & .590 \\
\hline Age at diagnosis-years & $65(58-73)$ & $66(57-75)$ & .553 \\
\hline Clinical-radiological diagnosis-n (\%) & $5(63)$ & $6(75)$ & .590 \\
\hline Smoking history-pack years & $12(1-23)$ & $10(1-19)$ & .400 \\
\hline UIP pattern on HRCT-n (\%) & $4(50)$ & $3(38)$ & .614 \\
\hline Prior treatment received steroids $-\mathrm{n}(\%)$ & $1(13)$ & $2(25)$ & .522 \\
\hline \multicolumn{4}{|l|}{ IPF treatment at baseline } \\
\hline Oxygen therapy at baseline $-\mathrm{n}(\%)$ & $3(38)$ & $2(25)$ & .590 \\
\hline Hypertension-n (\%) & $2(25)$ & $1(13)$ & .522 \\
\hline Diabetes mellitus-n (\%) & $1(13)$ & $2(25)$ & .522 \\
\hline GERD-n (\%) & $2(25)$ & $1(13)$ & .522 \\
\hline Emphysema-n (\%) & $2(25)$ & $1(13)$ & .522 \\
\hline \multicolumn{4}{|c|}{$\begin{array}{l}\text { Values are expressed as numbers and (\%) or median and ranges as appropriate. To compare } \\
\text { demographic data and baseline clinical characteristics between two groups, Chi square test for } \\
\text { categorical variables. }\end{array}$} \\
\hline
\end{tabular}

\subsection{Pirfenidone combined with DM mitigates pulmonary function decline in IPF patients}

Pulmonary function tests (PFTs) were performed before medication administration, and again at 6 months and 1 year. Before antifibrotic treatment and 6 months later, there were no significant differences 
in the PFTs between the pirfenidone group and the pirfenidone-DM group (Table 2). Some PFT indexes of the two groups showed an overall downward trend, but DLCO\%pred was not different between the two groups 1 year later (Fig. 2E). However, the pulmonary function tests, including FVC, FVC\%pred, FEV1, and FEV1\%pred in the pirfenidone-DM group were significantly better at 1 year after treatment than those in the pirfenidone group $(P<0.05)(F i g .2 A-D)$. Therefore, pirfenidone combined with DM significantly mitigated the pulmonary function decline compared with pirfenidone alone in patients with IPF. 
Table 2

Changes pulmonary function tests (PFTs).

\begin{tabular}{|c|c|c|c|}
\hline PFT & Pirfenidone $(\mathrm{N}=8)$ & Pirfenidone + DM $(\mathrm{N}=8)$ & $P *$ \\
\hline \multicolumn{4}{|l|}{$\mathrm{FVC}-\mathrm{L}$} \\
\hline at diagnosis & $3.03(2.60-3.59)$ & $2.97(2.40-3.65)$ & .999 \\
\hline 6 months & $2.42(2.10-3.14)$ & $2.78(1.88-3.68)$ & .744 \\
\hline 1 year & $1.79(1.55-2.18)$ & $2.60(1.68-3.49)$ & .038 \\
\hline \multicolumn{4}{|l|}{ FVC -\% pred. } \\
\hline at diagnosis & $79.03(56.40-90.30)$ & $76.57(59.60-93.30)$ & .999 \\
\hline 6 months & $70.73(47.30-80.60)$ & $75.25(54.60-93.50)$ & .999 \\
\hline 1 year & $54.70(41.30-61.50)$ & $73.73(53.50-91.20)$ & .044 \\
\hline \multicolumn{4}{|l|}{ FEV1 -L } \\
\hline at diagnosis & $2.34(1.59-3.16)$ & $2.36(1.68-2.89)$ & .999 \\
\hline 6 months & $2.10(1.46-2.66)$ & $2.40(1.73-3.02)$ & .952 \\
\hline 1 year & $1.61(1.13-1.98)$ & $2.39(1.76-3.02)$ & .044 \\
\hline \multicolumn{4}{|c|}{ FEV1 -\% pred. } \\
\hline at diagnosis & $83.15(57.50-94.20)$ & $86.15(68.20-110.70)$ & .999 \\
\hline 6 months & $76.75(53.90-89.30)$ & $86.67(57.80-116.30)$ & .906 \\
\hline 1 year & $64.28(45.60-73.30)$ & $88.28(55.40-118.90)$ & .049 \\
\hline \multicolumn{4}{|c|}{ DLCO -\% pred. } \\
\hline at diagnosis & $62.87(42.60-78.40)$ & $67.57(47.50-75.80)$ & .999 \\
\hline 6 months & $55.43(33.10-82.70)$ & $61.70(48.00-83.70)$ & .999 \\
\hline 1 year & $45.75(20.10-77.60)$ & $59.10(40.50-8450)$ & .508 \\
\hline \multicolumn{4}{|c|}{$\begin{array}{l}\text { Values are expressed as median and ranges as appropriate. FVC, Forced Vital Capacity; FVC\%pred, } \\
\text { forced vital capacity percent predicted; FEV1, forced expiratory volume in one second; FEV1\%pred, } \\
\text { forced expiratory volume in one second percent predicted; DLCO\%pred, diffusing capacity of the lung } \\
\text { for carbon monoxide percent predicted. }\end{array}$} \\
\hline
\end{tabular}

\subsection{Pirfenidone combined with DM ameliorated pulmonary imaging scores of patients with IPF}


The interobserver agreement between the two radiologists with regard to changes in alveolar score, reticulation, and honeycombing was good (Cohen's kappa $=0.74$ for alveolar score, $\mathrm{k}=0.78$ for reticulation, $\mathrm{k}=0.72$ for honeycombing). There was no significant difference in HRCT alveolar score, interstitial score or honeycomb score between the two groups of patients before treatment (Table 3). After 1 year of antifibrotic treatment, HRCT showed that the alveolar and interstitial scores of patients in the pirfenidone-DM group were markedly improved compared to those in the pirfenidone group $(P=0.035, P$ $=0.037$, respectively) (Table 3 , Fig. 3A, B), although the honeycomb scores remained similar between the two groups $(P=0.999)$ (Table 3, Fig. $3 C$ ). Thus, pirfenidone combined with DM significantly ameliorated the pulmonary imaging scores of patients with IPF. The patients' chest HRCT showed alleviated ground glass opacities and reticulation after 1 year administration of pirfenidone plus DM (Fig. 4).

Table 3

Changes in CT characteristics.

\begin{tabular}{|llll|}
\hline CT & Pirfenidone (N= 8) & Pirfenidone + DM (N= 8) & $P^{*}$ \\
\hline Alveolar score-\%(GGO) & $31.67(13.33-53.33)$ & $33.33(6.67-66.67)$ & .965 \\
\hline Alveolar score-\% 1 year & $37.50(20.00-53.33)$ & $20.00(0.00-26.67)$ & .035 \\
\hline Interstitial score-\% (reticulation) & $40.83(33.33-53.33)$ & $55.0(33.33-86.67)$ & .124 \\
\hline Interstitial score-\% 1 year & $56.67(40.00-66.67)$ & $38.33(13.33-53.33)$ & .037 \\
\hline Honeycomb score-\%(honeycombing) & $32.50(20.00-46.67)$ & $30.84(13.33-46.67)$ & .976 \\
\hline Honeycomb score-\% 1 year & $29.17(6.67-60.00)$ & $28.33(0.00-60.00)$ & .999 \\
\hline Values are expressed as numbers and (\%) or median and ranges as appropriate. & \\
\hline
\end{tabular}

\subsection{DM did not increase the adverse reactions of pirfenidone}

Two patients ( 1 from the pirfenidone group and 1 from the pirfenidone-DM group) had gastrointestinal reactions, and 1 patient from the pirfenidone group felt slight dizziness. There was no between-group difference in the risk of adverse reactions (t-test, $P>0.5$ ). The liver and kidney function and routine blood tests of the patients showed no significant differences between the two groups, and no significant changes were observed before and after medication $(P>0.5)$.

\section{Discussion}

In patients with IPF, after individual treatment and various immunotherapies, the epithelial cells and blood vessels in the lung are damaged, resulting in cell destruction, uncontrolled repair, and the development of progressive pulmonary fibrosis. Combining various hypotheses, when exposed to pathogenic factors, once fibrosis has formed, anti-fibrosis treatment should be initiated. Previously, progressive fibrosis was 
mostly classified as ordinary pulmonary fibrosis. Once fibrosis occurs, immunosuppression is no longer effective, and antifibrosis therapy is needed to stop the decline in lung function caused by pulmonary fibrosis. Pirfenidone was the first therapeutic drug to be approved for treating idiopathic pulmonary fibrosis. Pirfenidone was recommended conditionally for treatment as antifibrotic therapy of IPF patients in clinical practice guidelines in 2015 [8] because it can only moderately slow their decline of lung function. Since then, little clinical progress has been made. However, in the current study, DM significantly potentiated the antifibrotic efficacy of pirfenidone not only in mice induced by bleomycin but also in patients with IPF.

Researchers have proposed that the core pathway that mediates fibrosis may be a better target to develop drugs for anti-fibrosis in multiple organ systems [21] and that addressing the age-related redox imbalances has been recognized as one of these core approaches [22]. During the development of IPF, oxidative stress refers to the imbalance between the production and removal of oxygen free radicals in the body or cells, which leads to an increase in reactive oxygen species (ROS). When there is too much ROS production or insufficient antioxidant capacity in the body, excessive ROS exists in tissues or cells, which can induce oxidative stress and inevitably lead to lung tissue damage and remodelling[23].

DM is derived from non-opioid morphinans. As a widely used and safe over-the-counter cough suppressant, it has a clinical use history of more than 50 years[24]. Structurally, it belongs to morphine drugs. In view of its right-handed structure, DM has a weak affinity for opioid receptors, and it cannot really induce addiction. DM can specifically bind to the phosphorylated form of the subunit gp91 in NOX2, thereby inhibiting the catalytic activity of NOX2 and inhibiting the production of ROS. DM, as a broad and safe antitussive drug, has been found to have obvious anti-inflammatory and antioxidative effects in recent years. Previous studies have confirmed that DM antagonizes inflammation in the nervous system and prevents neurodegeneration. Moreover, DM significantly reduces the production of superoxide in neutrophils. We speculated that DM might play an antifibrotic role and enhance the antifibrotic effect of PFD through these mechanisms. To explore the antifibrotic effect of a combination of pirfenidone and DM on IPF, we conducted this study and found that a combination of the two can significantly improve pulmonary fibrosis. In animal studies, compared with other groups, pirfenidone combined with DM improved bleomycin-induced pulmonary fibrosis, as indicated by decreased proliferation of the alveolar epithelium, the production of fibroblasts, and the levels of hydroxyproline. In clinical studies, we observed that pirfenidone combined with DM could improve the PFT of patients, delay the reduction of FEV1 and FVC, and mitigate the ground glass opacities and reticulation of HRCT.

As a functional examination, PFTs mainly reflect the damage to lung ventilation and ventilation function caused by disease. Although FEV1 and FVC are affected by many factors, such as alveolar elasticity and airway resistance during expiration, pirfenidone combined with DM in this study was better than pirfenidone treatment in delaying the decline in FEV1 and FVC in IPF patients. Pulmonary diffusion function depends on the gas diffusion area in the lung, the thickness of the alveolar capillary membrane and the blood flow in the lung. The deterioration of lung diffusion during IPF is aggravated by the excessive proliferation of fibroblasts and the continuous deposition of extracellular matrix in the alveoli 
and lung interstitium accompanied by pulmonary vascular remodelling and occlusion. In terms of diffusion function, DLCO\%pred after treatment was not significantly different between the two groups before and after treatment, although pirfenidone plus DM appeared to slightly increase DLCO\%pred compared with pirfenidone alone.

High-resolution computed tomography (HRCT) plays a central role in diagnosing and staging the severity of IPF[17, 25]. HRCT can accurately predict the presence of UIP when features of honeycombing in a basilar, peripheral distribution are present $[2,26]$. The overall extent of lung fibrosis on CT (a combination of reticulation and honeycombing) is a proxy of disease severity and is a strong independent predictor of mortality in patients with IPF [18]. In our study, pirfenidone combined with DM antifibrosis therapy slowed the decline in FVC and FEV1 in patients with IPF compared with single-agent pirfenidone treatment. After 1 year of treatment, it had a significant therapeutic effect on pulmonary function testing, but we also observed that it had no significant effect in improving the HRCT of the IPF patients' honeycombs. The DLCO\% of the IPF patients decreased, and the pathological changes on HRCT mainly showed reticulation and honeycombing, which is consistent with the good correlation between reticulation and honeycombing and DLCO\%.

Adverse reactions to pirfenidone are common and mainly include gastrointestinal manifestations, skin diseases and dizziness [27]. The combination of pirfenidone and DM was well tolerated by patients in this study, and DM did not increase the side effects of pirfenidone. Add-on therapy containing DM has potent effects in many clinical settings. NEW YORK, Dec. 08, 2020, Very recently, Axsome Therapeutics announced positive results from a phase II clinical trial of AXS-05 in patients with major depressive disorder. After receiving AXS-05 (45 mg dextromethorphan-105 mg bupropion) twice daily for up to 12 months, the patients experienced a rapid reduction of suicidal ideation and functional improvement with good safety.

In this study, DM alone showed an antifibrotic effect and enhanced the antifibrotic mechanism of PFD. We have not conducted research on the underlying mechanism. The sample size of this clinical study is relatively small, which is not sufficientto provide an accurate basis for guiding clinical treatment, so additional and larger-scale RCTs are needed for verification.

\section{Conclusions}

DM significantly potentiates the antifibrotic effectiveness of pirfenidone in a mouse IPF model and patients with IPF and does not increase the side effects of pirfenidone. The efficacy and safety of the combination of pirfenidone and DM for patients with IPF warrants further verification by the double-blind randomized controlled trial (RCT). We also need to study the mechanism of DM synergistic anti-fibrosis.

\section{Abbreviations}


IPF: idiopathic pulmonary fibrosis; PFD: pirfenidone; DM: dextromethorphan; FEV1: forced expiratory volume in one second; FEV1\%pred: forced expiratory volume in one second as percentage of predicted volume; FVC: forced vital capacity; FVC\%pred: forced vital capacity as percentage second as percentage of predicted volume; DLCO: diffusing capacity of the lungs for carbon monoxide; ROS:reactive oxygen species

\section{Declarations}

\section{Acknowledgements}

Our research complies with the ARRIVE guidelines. The authors thank the patients for participating and donating samples to make this research possible. Thanks to all hospitals and institutions that participated and provided data.

\section{Funding}

This work was supported by the grants from the National Natural Science Foundation of China (No.81970083, 81270144, 81570084 and 30800507).

\section{Availability of data and materials}

The datasets used and/or analyzed during the current study are available from the corresponding author on reasonable request.

\section{Authors' contributions}

$\mathrm{LN}$ and HJ performed research, collected data and wrote the manuscript. WY performed statistical analysis. DY collected data. JH analyzed and interpreted data. FJ and NW designed research and performed research. All authors read and approved the final manuscript.

\section{Ethics approval and consent to participate}

Experiments in mice were approved by the local committee for animal welfare. For the human study, the protocol has been approved by the Ethics Committee of Tianjin Medical University General Hospital, China (No. IRB2020-YX-031-01). This study is registered for Chinese Clinical Trial Registry (Registration Number: ChiCTR1900023727). Written informed consent for participation in the study was obtained from all the patients. 


\section{Consent for publication}

Not applicable.

\section{Competing interests}

The authors declare that they have no competing interests.

\section{References}

1. Lederer DJ, Martinez FJ: Idiopathic Pulmonary Fibrosis. N Engl J Med 2018, 378: 1811-1823.

2. American Thoracic Society/European Respiratory Society: International multidisciplinary consensus classification of the idiopathic interstitial pneumonias. Am J Respir Crit Care Med 2002, 165: 277304.

3. Chakraborty S, Chopra P, Ambi SV, Dastidar SG, Ray A: Emerging therapeutic interventions for idiopathic pulmonary fibrosis. Expert Opin Inverstig Drugs 2014, 23: 893-910.

4. Kahloon RA, Xue J, Bhargava A, Csizmadia E, Otterbein L, Kass DJ, Bon J, Soejima M, Levesque MC, Lindell KO, et al: Patients with idiopathic pulmonary fibrosis with antibodies to heat shock protein 70 have poor prognoses. Am J Respir Crit Care Med 2013, 187:768-775.

5. Ishikawa F, Kaneko E, Sugimoto T, Ishijima T, Wakamatsu M, Yuasa A, Sampei R, Mori K, Nose K, Shibanuma M: A mitochondrial thioredoxin-sensitive mechanism regulates TGF-beta-mediated gene expression associated with epithelial-mesenchymal transition. Biochem Biophys Res Commun 2014, 443:821-827.

6. Manoury B, Nenan S, Leclerc O, Guenon I, Boichot E, Planquois JM, Bertrand CP, Lagente V: The absence of reactive oxygen species production protects mice against bleomycin-induced pulmonary fibrosis. Respir Res 2005, 6:11.

7. Oku H, Shimizu T, Kawabata T, Nagira M, Hikita I, Ueyama A, Matsushima S, Torii M, Arimura A: Antifibrotic action of pirfenidone and prednisolone: different effects on pulmonary cytokines and growth factors in bleomycin-induced murine pulmonary fibrosis. Eur J Pharmacol 2008, 590: 400408.

8. Raghu G, Rochwerg B, Zhang Y, Garcia CA, Azuma A, Behr J, Brozek JL, Collard HR, Cunningham W, Homma S, et al: An Official ATS/ERS/JRS/ALAT Clinical Practice Guideline: Treatment of Idiopathic Pulmonary Fibrosis. An Update of the 2011 Clinical Practice Guideline. Am J Respir Crit Care Med 2015, 192:e3-19.

9. Noble PW, Albera C, Bradford WZ, Costabel U, Glassberg MK, Kardatzke D, King TE, Lancaster L, Sahn SA, Szwarcberg J, et al: Pirfenidone in patients with idiopathic pulmonary fibrosis (CAPACITY): two randomised trials. The Lancet 2011, 377:1760-1769. 
10. Ang ET, Wong PT, Moochhala S, Ng YK: Cytokine changes in the horizontal diagonal band of Broca in the septum after running and stroke: a correlation to glial activation. Neuroscience 2004, 129:337347.

11. Lee SY, Chen SL, Chang YH, Chu CH, Chen SH, Chen PS, Huang SY, Tzeng NS, Wang LJ, Lee IH, et al: A placebo-controlled trial of dextromethorphan as an adjunct in opioid-dependent patients undergoing methadone maintenance treatment. Int J Neuropsychopharmaco/2015, 18:pyv008.

12. Pu B, Xue $Y$, Wang Q, Hua C, Li X: Dextromethorphan provides neuroprotection via anti-inflammatory and anti-excitotoxicity effects in the cortex following traumatic brain injury. Mol Med Rep 2015, 12:3704-3710.

13. Li MH, Luo YH, Lin CF, Chang YT, Lu SL, Kuo CF, Hong JS, Lin YS: Dextromethorphan efficiently increases bactericidal activity, attenuates inflammatory responses, and prevents group a streptococcal sepsis. Antimicrob Agents Chemother 2011, 55:967-973.

14. Li G, Liu Y, Tzeng NS, Cui G, Block ML, Wilson B, Qin L, Wang T, Liu B, Liu J, Hong JS: Protective effect of dextromethorphan against endotoxic shock in mice. Biochem Pharmacol 2005, 69:233240.

15. Jiang D, Liang J, Hodge J, Lu B, Zhu Z, Yu S, Fan J, Gao Y, Yin Z, Homer R, et al: Regulation of pulmonary fibrosis by chemokine receptor CXCR3. Journal of Clinical Investigation 2004, 114:291299.

16. Adamson IY, Bowden DH: The pathogenesis of bleomycin-induced pulmonary fibrosis in mice. Am J Pathol 1974, 77:185-197.

17. Raghu G, Remy-Jardin M, Myers JL, Richeldi L, Ryerson CJ, Lederer DJ, Behr J, Cottin V, Danoff SK, Morell F, et al: Diagnosis of Idiopathic Pulmonary Fibrosis. An Official ATS/ERS/JRS/ALAT Clinical Practice Guideline. Am J Respir Crit Care Med 2018, 198:e44-e68.

18. Best AC,Meng J,Lynch AM,Bozic CM,Miller D,Grunwald GK,Lynch DA: Idiopathic pulmonary fibrosis: Physiologic tests, quantitative $\mathrm{CT}$ indexes, and $\mathrm{CT}$ visual scores as predictors of mortality. Radiology 2008, 246: 935-940.

19. Kazerooni EA, Martinez FJ, Flint A, Jamadar DA, Gross BH, Spizarny DL, Cascade PN, Whyte RI, Lynch JP, Toews G: Thin-section CT obtained at 10-mm increments versus limited three-level thin-section CT for idiopathic pulmonary fibrosis: correlation with pathologic scoring. AJR Am J Roentgenol 1997, 169: 977-983.

20. Balestro E, Cocconcelli E, Giraudo C, Polverosi R, Biondini D, Lacedonia D, Bazzan E, Mazzai L, Rizzon G, Lococo S, et al: High-Resolution CT Change over Time in Patients with Idiopathic Pulmonary Fibrosis on Antifibrotic Treatment. J Clin Med 2019, 8.

21. Mehal WZ, Iredale J, Friedman SL: Scraping fibrosis: Expressway to the core of fibrosis. Nature Medicine 2011, 17:552-553.

22. Liepelt $A$, Tacke F: Healing the scars of life-targeting redox imbalance in fibrotic disorders of the elderly. Ann Trans/ Med 2015, 3:S13. 
23. Fois AG, Paliogiannis P, Sotgia S, Mangoni AA, Zinellu E, Pirina P, Carru C, Zinellu A: Evaluation of oxidative stress biomarkers in idiopathic pulmonary fibrosis and therapeutic applications: a systematic review. Respir Res 2018, 19:51.

24. Chou YC, Liao JF, Chang WY, Lin MF, Chen CF: Binding of dimemorfan to sigma-1 receptor and its anticonvulsant and locomotor effects in mice, compared with dextromethorphan and dextrorphan. Brain Res 1999, 821: 516-519.

25. Lynch DA, Sverzellati N, Travis WD, Brown KK, Colby TV, Galvin JR, Goldin JG, Hansell DM, Inoue Y, Johkoh T, et al: Diagnostic criteria for idiopathic pulmonary fibrosis: a Fleischner Society White Paper. Lancet Respir Med 2018, 6: 138-153.

26. Hunninghake GW, Zimmerman MB, Schwartz DA, King TE, Lynch J, Hegele R, Waldron J, Colby T, Müller N, Lynch D, et al: Utility of a lung biopsy for the diagnosis of idiopathic pulmonary fibrosis. $A m$ J Respir Crit Care Med 2001, 164: 193-196.

27. Noble PW, Albera C, Bradford WZ, Costabel U, Glassberg MK, Kardatzke D, King TE, Lancaster L, Sahn SA, Szwarcberg J, et al: Pirfenidone in patients with idiopathic pulmonary fibrosis (CAPACITY): two randomised trials. The Lancet 2011, 377:1760-1769.

\section{Figures}


HE

Control

$\mathrm{BLM}+\mathrm{NaCl}$

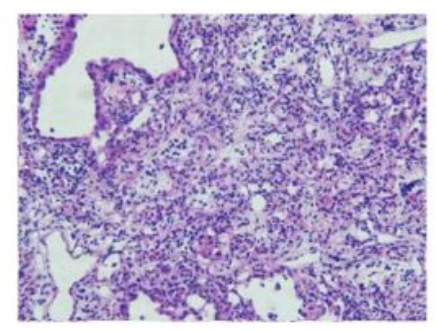

BLM+DM

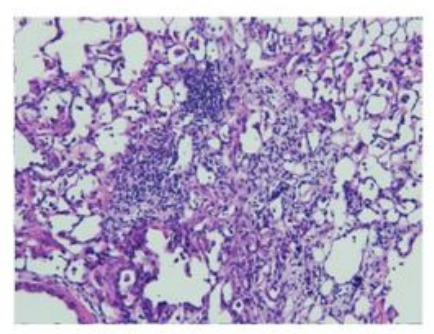

BLM+PFD

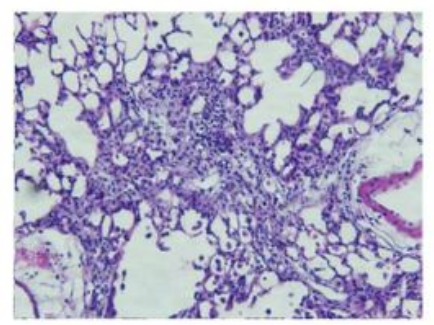

BLM+PFD+DM

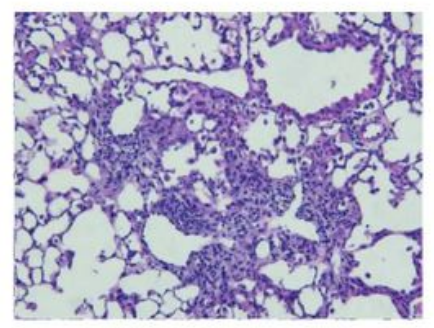

Masson
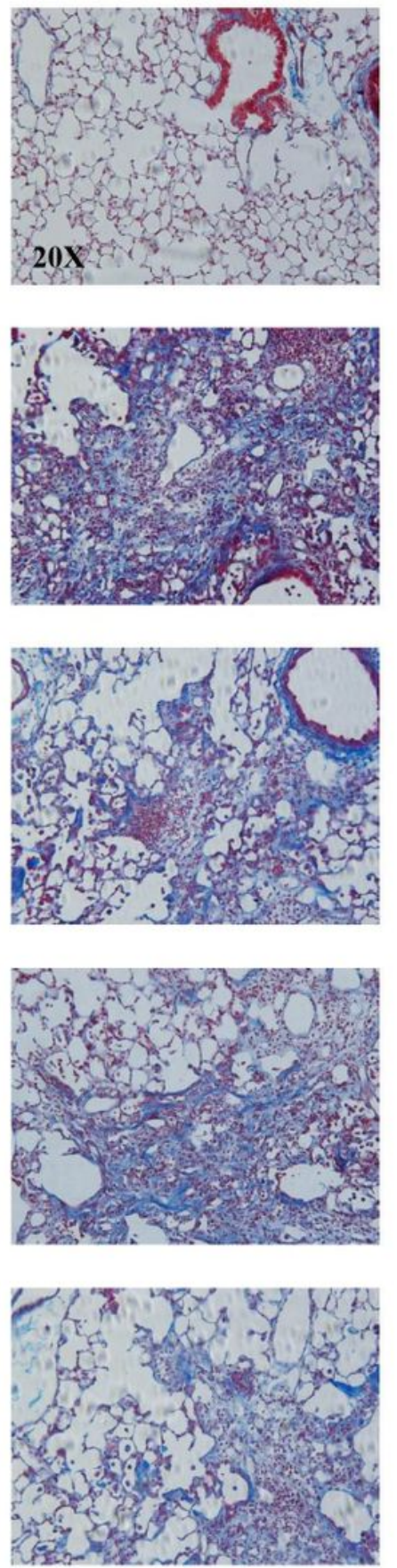

B

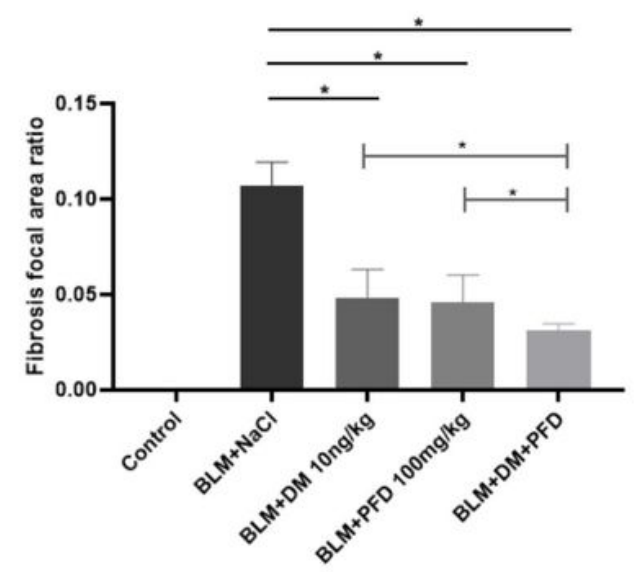

C

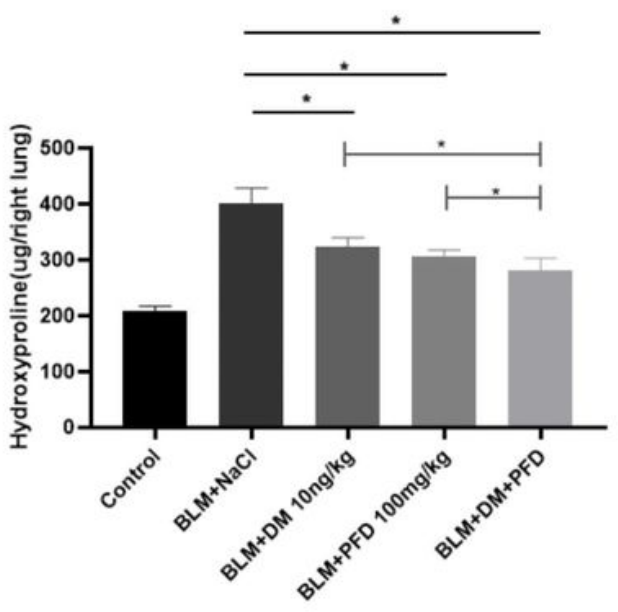

\section{Figure 1}

Pirfenidone combined with DM reduce bleomycin-induced pulmonary fibrosis in mice at D21. (A) As indicated by $H \& E$ staining of lung sections, the intratracheal injection of bleomycin led to the destruction of normal pulmonary architecture, the prominently thickening of alveolar septum and the mass filling of fibrous tissue. As illustrated by Masson's trichrome staining, the deposition of collagen fibers was largely increased in bleomycin-induced lung injury. Pirfenidone combined with DM alleviated pulmonary pathological changes and reduced the production of collagen. Pirfenidone plus DM significantly 
decreased fibrosis focal area ratio (B) and hydroxyproline measurement in the lungs (C). Data are the mean \pm SD. Statistical analyses were performed by an ANOVA one-way test with Tukey's test for comparisons between groups. ${ }^{*}, \mathrm{P}<0.05$.

A

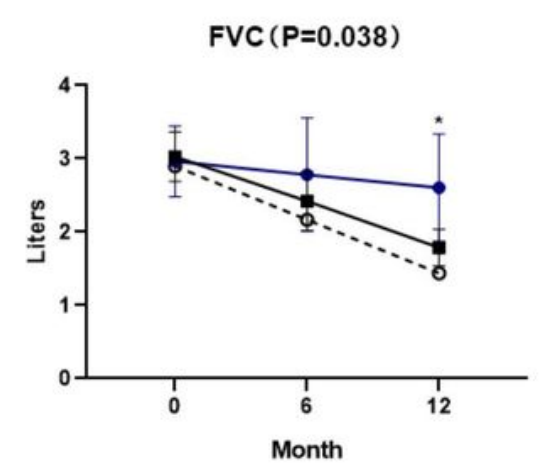

$\mathrm{C}$

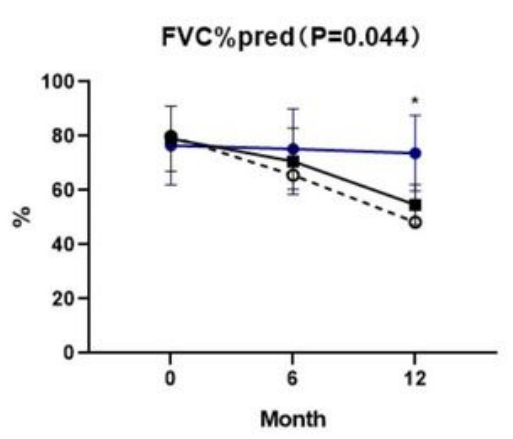

$\mathrm{E}$

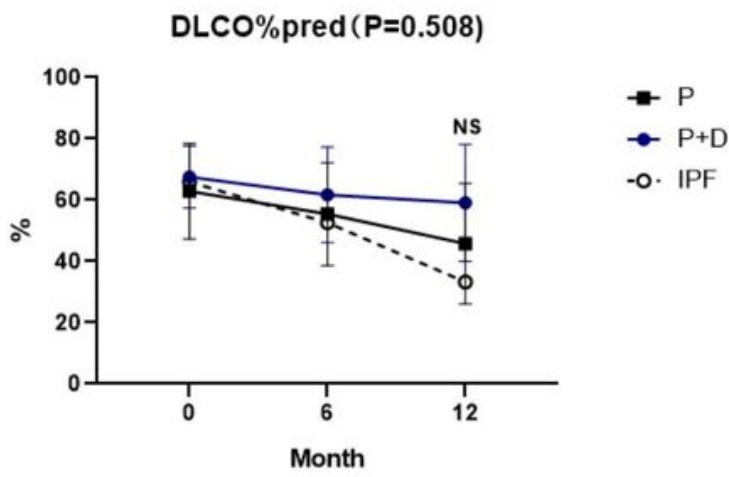

$\mathrm{B}$

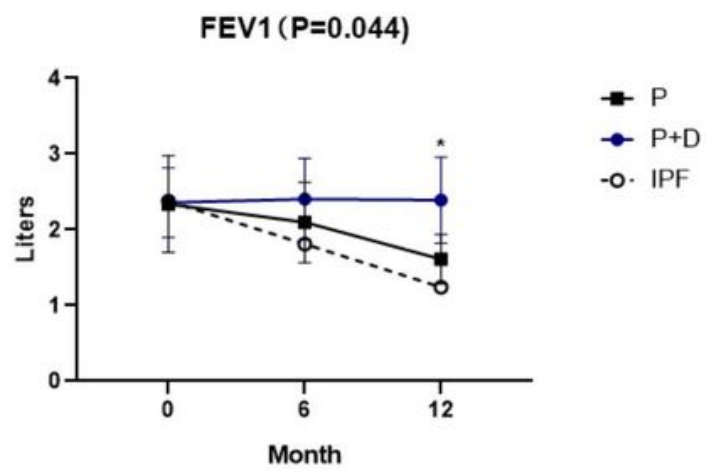

$\mathrm{D}$

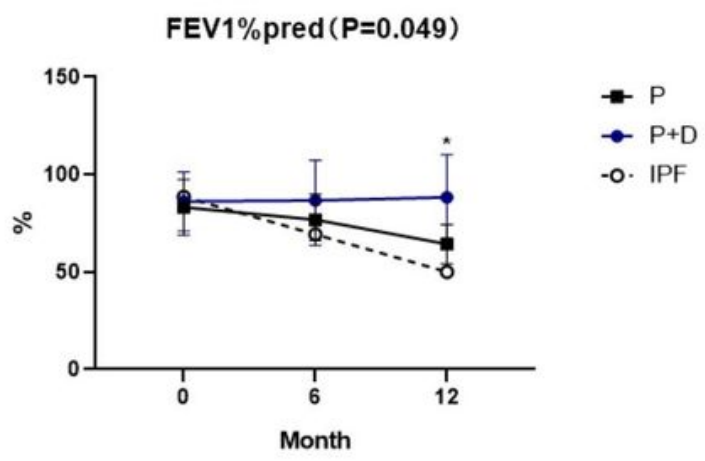

Figure 2

PFT indexes of two groups of patients. Except DLCO\%pred, the comparison of FVC, FEV1, FVC\%pred, FEV1\%pred showed statistical differences between the two groups $(P F V C=0.038, P F E V 1=0.044$, 
PFVC\%pred=0.044, and PFEV1\%pred=0.049). Reference baseline for lung function in IPF patients comes from other studies[20]. ${ }^{*} \mathrm{p}<0.05$ and NS (no significance) compared to pirfenidone group. Two-way ANOVA followed by Bonferroni post hoc multiple comparison test was performed.
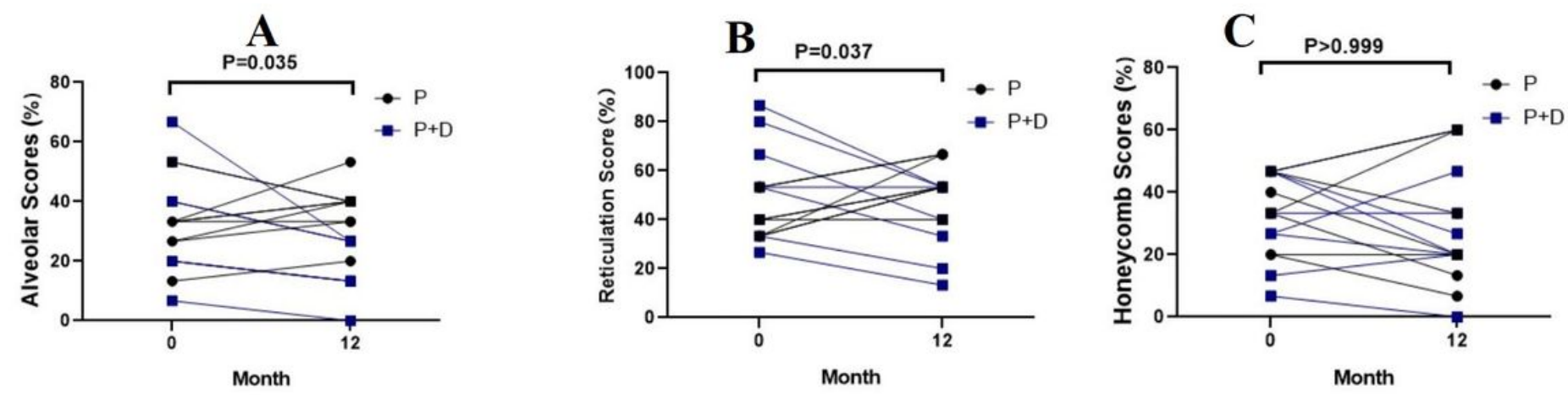

\section{Figure 3}

Alveolar score, interstitial score, and honeycomb score of patients treated with pirfenidone $(P)$ or pirfenidone plus dextromethorphan $(P+D)$. Comparison of Alveolar score, Interstitial score and Honeycomb score of HRCT between two groups of patients before and after treatment. Two-way ANOVA followed by Bonferroni post hoc multiple comparison test was performed.

A

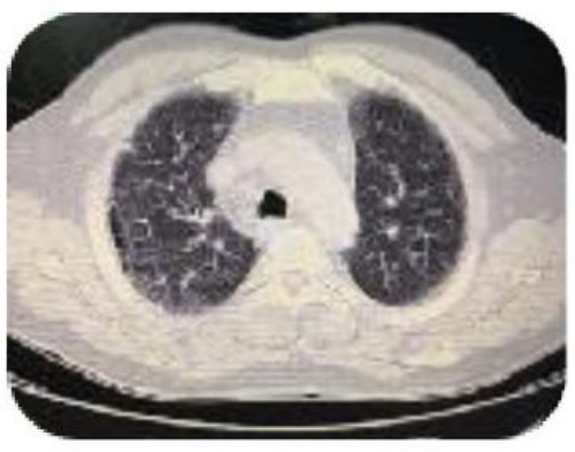

$\mathrm{D}$

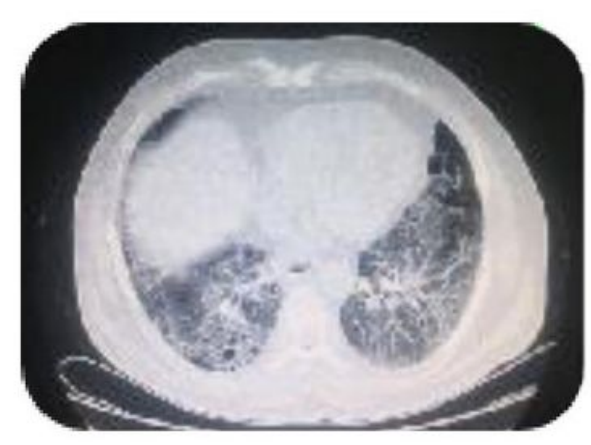

B

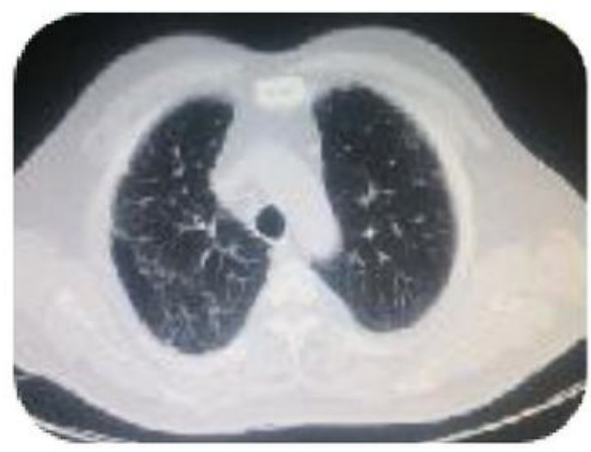

E

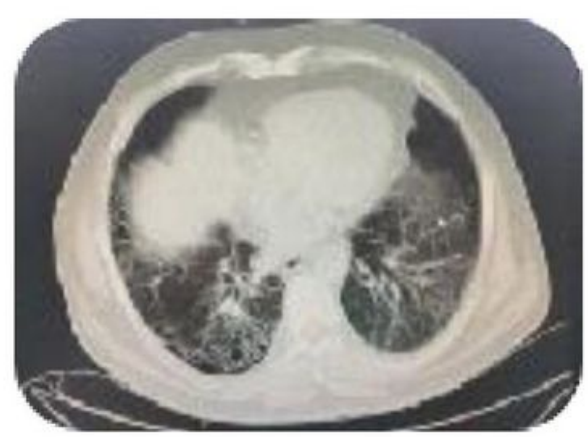

C

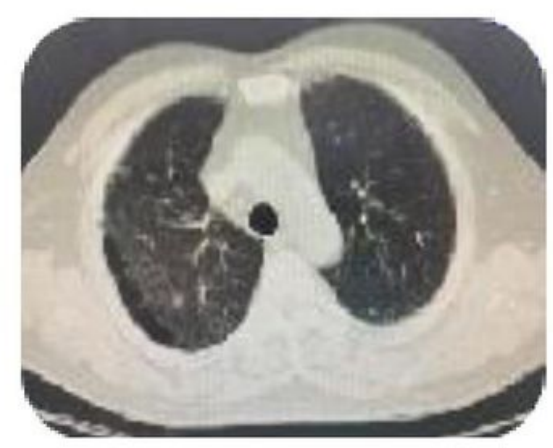

F

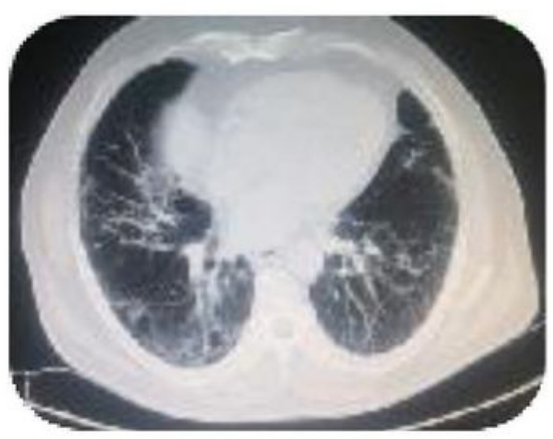

Figure 4 
Comparisons of HRCT images of a patient received pirfenidone and DM before and after treatment. Male, $61 \mathrm{y}$, with a history of pulmonary fibrosis for 4 years and a history of smoking. Initial HRCT (A and D), 6 months later $\mathrm{HRCT}(\mathrm{B}$ and $\mathrm{E})$, and one year later HRCT ( $\mathrm{C}$ and $\mathrm{F}$ ) displayed apparently less ground glass opacities, reticulation and honeycombing after treatment. 